\title{
FOOD ALLERGY AS A CAUSE OF ABDOMINAL PAIN
}

\author{
WILLIAM W. DUKE. M.D. \\ KANSAS CITY, MO.
}

Severe abdominal pain is a symptom which is never taken lightly by a careful physician. It often indicates a severe illness; in fact, often indicates an emergency. However, abdominal pain which may simulate in some respects the pain caused by serious abdominal disease is occasionally the result of hypersensitiveness to a food.

It has been known for a number of years that hypersensitiveness to foods may give rise to bronchial asthma and a condition which simulates hay fever; to urticaria, angioneurotic edema, purpura, eczema and other dermatoses; to dyspepsia, gastro-intestinal upsets associated with vomiting, diarrhea, gripping pains in the abdomen and mucous colitis; to an interesting syndrome of symptoms known as Henoch's purpura, and that with the latter conditions a patient may have severe abdominal pain. Abdominal pain occasionally occurs alone, however, or rather, it is occasionally the sole striking symptom of hypersensitiveness to a food. In this case, the real cause of pain may not be apparent, and an error in diagnosis can easily be made. It is this type of illness that I wish to discuss.

The condition is well illustrated by the case reports. The cases have been chosen from a larger number to illustrate typical histories of patients who have abdominal pain as the outstanding symptom of hypersensitiveness to a food. For this reason only such details as pertain to this subject will be related.

\section{HISTOR:}

The phenomenon of sensitiveness or hypersensitiveness to alien matter has been discussed in its relationship to infection and immunity for many years. A cutaneous reaction obtained by applying smallpox virus to a scarified area on the skin of an immune individual was described in detail by Jenner as early as 1789 . Magendie in 1839, and Flexner in 1894. apparently observed anaphylaxis in animals after inoculation with foreign material. Their observations were overlooked. however, and the subject did not attract much attention in medical literature until after the publications of Richet, in 1902. While experimenting with a toxin extracted from actinae, Richet found that animals inoculated with this poison became unusually sensitive to it-in fact. so sensitive that a second dose, too small to be harmful to normal 
animals, was sufficient to cause certain violent symptoms in animals which had previously been inoculated. This peculiar condition Richet termed anaphylaxis.

Following Richet's work, a number of valuable pioneer contributions were made by other observers working independently of Richet or along different lines; namely Arthus, in 1903, Pirquet and Schick, ir 1903, Otto, investigating an observation made by Theobald Smith; Rosenow and Anderson, Vaughan, Besredka, Gay and Southard, and others. A number of remarkable facts were disclosed, the more important of which may be summarized as follows: Certain animals (particularly the guinea-pig) when inoculated with an alien albumin may become sensitive to that particular albumin. After being sensitized, future inoculations, instead of being practically harmless, are likely to cause violent symptoms of anaphylaxis which frequently result in the death of the animal. It was discovered that sensitization occurs only after an incubation period of several weeks or months has elapsed, that sensitiveness once established is likely to persist for years, even throughout the life of an animal; that the condition can be transferred passively to other animals by an inoculation of serum from a sensitized animal, that it can be transmitted from mother to offspring; and that sensitiveness may be removed quickly and completely by inoculation with sublethal doses of the albumin to which the animal is sensitive.

It was discovered that sensitiveness is remarkably specific for the type of proteid used in producing the sensitive state. For example, animals inoculated with milk might become remarkably sensitive to further inoculations with milk but not sensitive to serum-even serum of the same species. Likewise, animals sensitized against egg albumin might become sensitive to the particular egg albumin used in making the inoculation but not sensitive to other egg albumins nor to the serum of the same animals. Animals would be more sensitive to egg albumin of closely related species than to the serum of the same animal.

In 1910, Meltzer made a unique contribution to our knowledge of the subject. Comparing the bronchial constriction of asthma with that found in animals dying in anaphylactic shock, he suggested that real bronchial asthma was nothing more nor less than a phenomenon of anaphylaxis. This interesting suggestion attracted a great deal of attention, and since then a number of additional conditions have been attributed to a disturbed function caused by a remarkable hypersensitive state in humans which resembles anaphylaxis in many respects, but which seems to be fundamentally a different phenomenon. This state is described by more recent writers under the broader term "allergy."

The more interesting symptoms of allergy mentioned by Coca, Cook and others are, hay-fever, bronchial asthma, chronic joint disease, 
urticaria, angioneurotic edema, certain eczemas, certain gastro-intestinal conditions with symptoms such as abdominal pain, indigestion, vomiting and diarrhea and a symptom complex which is generally described under the term Henoch's purpura.

Whereas anaphylaxis and allergy have features and symptoms in common, they seem to be fundamentally different phenomena. This fact has been brought out especially by Coca, who mentions the following striking differences between the two conditions.

First.-The exciting agent of anaphylaxis is always antigenic in character. The exciting agent of allergy may or may not be an antigenic substance. (For example, it may be a drug such as arsphenamin or acetylsalicylic acid.)

Second.-Although anaphylaxis may be transmitted from mother to offspring, it is not inheritable in the true sense of the word. It is always primarily an artificial condition induced by the introduction of antigenic substance into the body of some susceptible animal.

Allergy, on the other hand, is always based primarily on a natural inherited makeup. The sensitiveness classed as allergy does not in every instance appear to depend on previous contact with an exciting substance. An individual, for example, may have a violent allergic reaction when he comes in contact with the exciting substance apparently for the first time.

Third.-The phenomenon of desensitization which can quickly and invariably be brought about in animals with anaphylactic sensitiveness is entirely wanting in allergy. The state of reduced sensitiveness observed clinically after treatment of allergy by hypodermic injections of the exciting agent is never complete nor is it comparable with the above.

For a complete discussion of these many interesting phases of this subject the writings of Coca, Cook, Walker, Vaughan, Besredka, Wells and others should be consulted.

\section{REPORT OF CASES}

CASE 1.-Male, aged 32, with family and past history negative for hay-fever, asthma and other symptoms of allergy, had a severe illness at the age of 2 , due to bee stings. He remembers that since earliest childhood he has invariably experienced a severe nonradiating gripping pain in the epigastrium, associated with nausea and vomiting, every time he has eaten honey. He has frequently tried to eat honey, but found that it invariably caused a severe attack of abdominal pain. The pain would start about fifteen minutes after the ingestion of honey, and last about three hours.

Previous Illnesses.-The patient has always been well otherwise. He has been free from other digestive disturbance and has never noticed other symptoms of allergy either during the attacks described nor between attacks.

Physical Examination.- Negative, except for chronic ethmoiditis. A cutaneous test made by applying honey to a scarified area on the skin gave a typical urticarial wheal about $2 \mathrm{~cm}$. in diameter, which appeared in less than five minutes and disappeared after about one-half hour. 
The patient was unwilling to eat honey as a clinical test or to allow a small amount to be injected subcutaneousy.

CASE 2.-Patient, male, aged 37, with negative past history, while out camping about ten years ago missed two meals and then ate an entire cake of honey on an empty stomach without other food. He experienced no discomfort at the time. Two months afterward he observed that whenever he ate honey, even in small quantities, he invariably experienced a steady, severe pain in the epigastrium, which lasted several hours. This condition has persisted to the present time. The pain can be brought on at will by the eating of a small amount of honey.

Previous Illnesses.-The patient gives no further history of dyspepsia, pain. or symptoms of allergy, except those mentioned. His entire history and physical examination is negative, except as mentioned.

On ingestion of one teaspoonful of honey, as a clinical test he had epigastric pain which lasted one-half hour.

A cutaneous test made by applying honey to a small scarified area on the skin gave rise in four minutes to a typical itching wheal $0.5 \mathrm{~cm}$. in diameter, which persisted for one and one-half hours.

CASE 3.-Male, aged 25, having a family and past history negative for hayfever, asthma, dermatoses and other symptoms of allergy, ate shad roe from two to five times a week at lunch for a period of several months. After removing to another locality, he did not eat shad roe for a period of about two years. $\mathrm{He}$ then became subject to occasional attacks of severe nonradiating, steady pain in the epigastrium which would develop about fifteen minutes after a meal, and last from three to five hours. Occasionally, the pain was associated with nausea and vomiting. The pain was so severe as to completely incapacitate him for mental or physical work. Several attacks were followed by tenderness over the appendix. which lasted for about a week. The case was finally diagnosed recurrent appendicitis and the appendix was removed. A few adhesions about the appendix were found, together with several concretions in its lumen. Similar attacks of epigastric pain occurred after the appendix was removed. They differed from the former attacks only in the fact that they were not followed by tenderness in the right iliac fossa. Finally, it was discovered that the attacks occurred invariably after the eating of shad roe and that if the shad roe was eliminated from the diet entirely no such attacks occurred. The patient had not at any time had hives, asthma or other symptoms of allergy.

This sensitiveness has now persisted for more than ten years, but is not so marked as it was originally. The ingestion of shad roe at the present time causes discomfort in the abdomen and nausea, but not severe pain.

Physical Examination.-Disclosed nothing of interest in this connection, except a moderate grade of ptosis.

An intracutaneous injection (made recently) with an aqueous extract of shad roe gave rise to a cutaneous reaction about $0.5 \mathrm{~cm}$. in diameter.

CASE 4.-Patient, female, aged 45. Her father had autumnal pollen asthma until his death several years ago. Her brother had summer and autumnal hayfever and also eczema on the hands until he was forced to leave this climate.

Previous Illnesses.-The patient gives no history of hay-fever, asthma, eczema nor other symptoms of allergy. Eight years ago abscess of the appendix formed and was drained. Five years ago she had an infected gallbladder, which was removed. One year after this, for the first time in her life, she began having indigestion, the outspoken symptom of which was a severe steady pain in the epigastrium, which would come on every day about one-half hour after eating. and last from one to three hours. It was often associated with vomiting. She found by experience that the pain was relieved to a certain extent by lavage. The patient had been dieted and treated without relief for several months before coming under my observation. The history was otherwise negative or of no interest in this connection. 


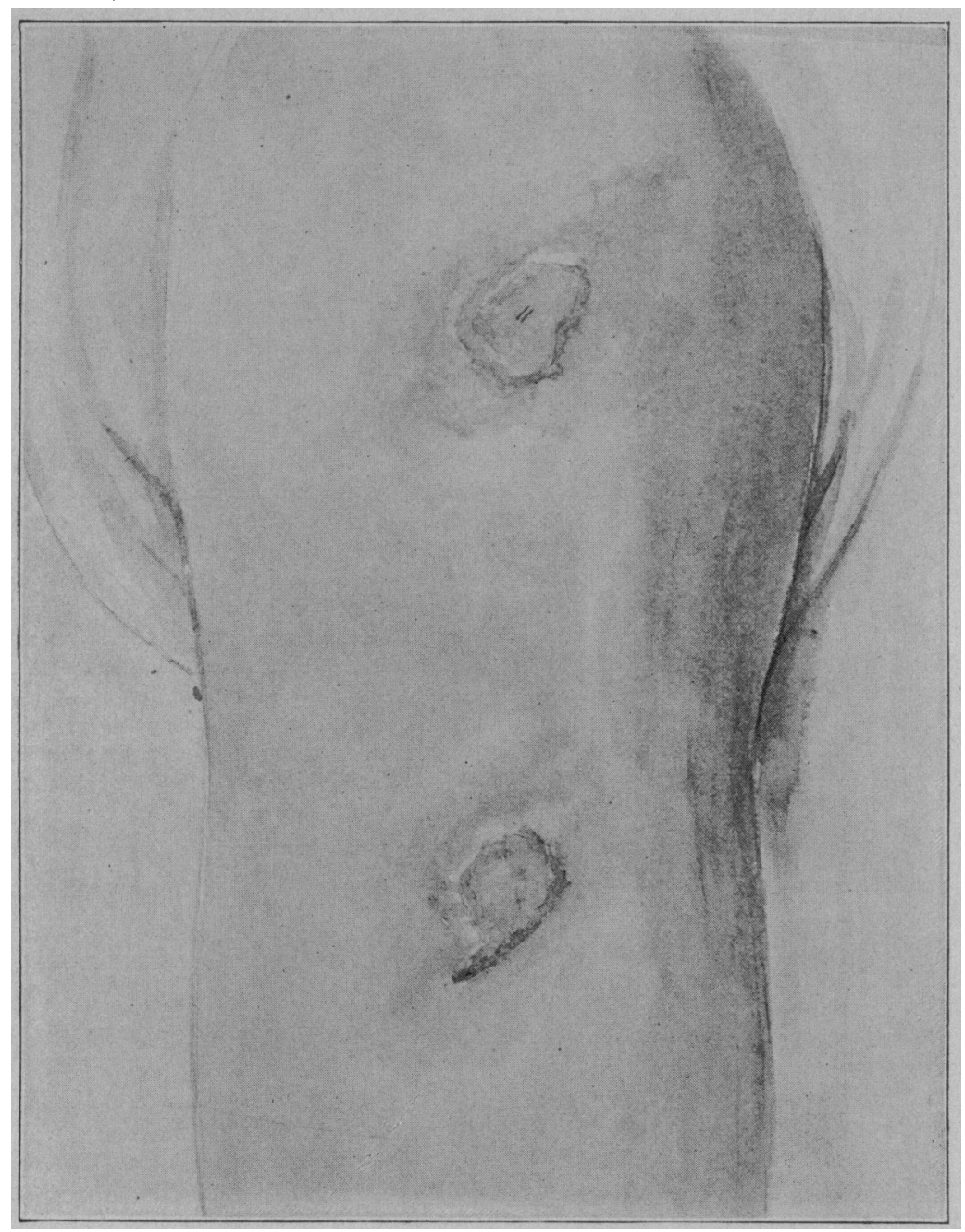

Fig. 1.-Positive cutaneous and intracutaneous tests. The two tests were made at the same time by applying honey (above) to a scarified area on the skin, and (below) by inoculating $0.02 \mathrm{mg}$. of honey intradermally. Both reactions appeared in less than four minutes, itched intensely and disappeared after about one and one-half hours. 
Physical Examination.-On examination was found a slight grade of oral sepsis, slight general abdominal tenderness, high grade ptosis, adhesions about the pyloric end of the stomach very evident by roentgen-ray examination and a bulb deformity, due evidently to adhesions. Examination was otherwise negative or of no interest.

Careful dietary and intracutaneous tests proved the patient to be sensitive to lactalbumin, beef proteid and egg white. Tests made by applying the above proteins in dry powdered form to a scarified area on the skin were negative. However, intracutaneous injection of the proteins mentioned gave rise in a few moments to typical wheals about $0.5 \mathrm{~cm}$. in diameter. Subcutaneous inoculations with small amounts of milk, egg white and beef serum, used subsequently in therapy, frequently gave rise to attacks of abdominal pain and vomiting similar to those of which the patient complained originally. The ingestion of a small amount of beef, milk and egg white, taken as a clinical test, in each instance gave rise to attacks of abdominal pain and vomiting, which came on within less than one-half hour and lasted from one to three hours. Other foods tested in this way caused little or no distress when she was on a diet free of egg, milk and beef proteins.

The patient was treated with subcutaneous inoculations of beef, milk and egg white (increasing from $0.001 \mathrm{mg}$. to $1 \mathrm{mg}$.) over a period of two months. She did not at any time during this treatment have hives, asthma, or other cutaneous or respiratory symptoms of allergy. A number of reactions, however, confined her to bed several hours with abdominal pain, vomiting and general malaise.

At the end of two months her sensitiveness had been reduced to such an extent that she was tolerant of the small amounts of milk and eggs used in the cooking of ordinary foods, so that she has been able since treatment (now two years) to live in comfort on an ordinary diet when she simply avoids the drinking of considerable amounts of milk and the eating of eggs or beef.

Intracutaneous tests with lactalbumin, beef proteins and egg white at the present time give definitely positive reactions.

CASE 5.-A man, aged 44, with uninteresting family and past history, began having dyspepsia and attacks of abdominal pain about six years before coming under observation. His dyspepsia had been practically unremitting during this entire period. He was subject to attacks of pain in the epigastrium, which would come on from two to four hours after eating, and last from six to thirtysix hours. At times, the pain was so severe as to completely incapacitate him. He was subject also to dyspepsia, the symptoms consisting of discomfort in the epigastrium, sour eructations, bloating and occasionally nausea, vomiting and diarrhea. He was subject to hives early in his illness. After these had persisted off and on for several months, he discovered that he coud keep himself relatively free of hives if he eliminated milk from his diet. Before coming under my observation he had discovered that he felt better and had less abdominal pain and dyspepsia when he avoided both beef and milk.

Physical Examination.--The subsequent course of the disease disclosed only three pathologic conditions of interest, namely, stone in the left ureter, gallstones and sensitiveness to milk and to beef serum. Intracutaneous tests with whole milk and beef serum gave rise to characteristic wheals about $1 \mathrm{~cm}$. in diameter.

The patient was put on a diet free of milk and beef. While on this diet he seemed very comfortable. While on a beef and milk free diet he was able to eat without trouble such foods as green vegetables, condiments, nuts, etc., which had previously caused discomfort. He was finally given a meal containing a little rare meat as a test. He experienced pain after this meal, which started after about six hours, and lasted about thirty-six hours. He refused to try further experiments of the sort.

The patient was then given a course of inoculations with beef serum and skimmed milk (from $0.001 \mathrm{mg}$. to $1 \mathrm{mg}$.) over a period of six. weeks. At the end of this time he was able to take small amounts of beef or milk by mouth 


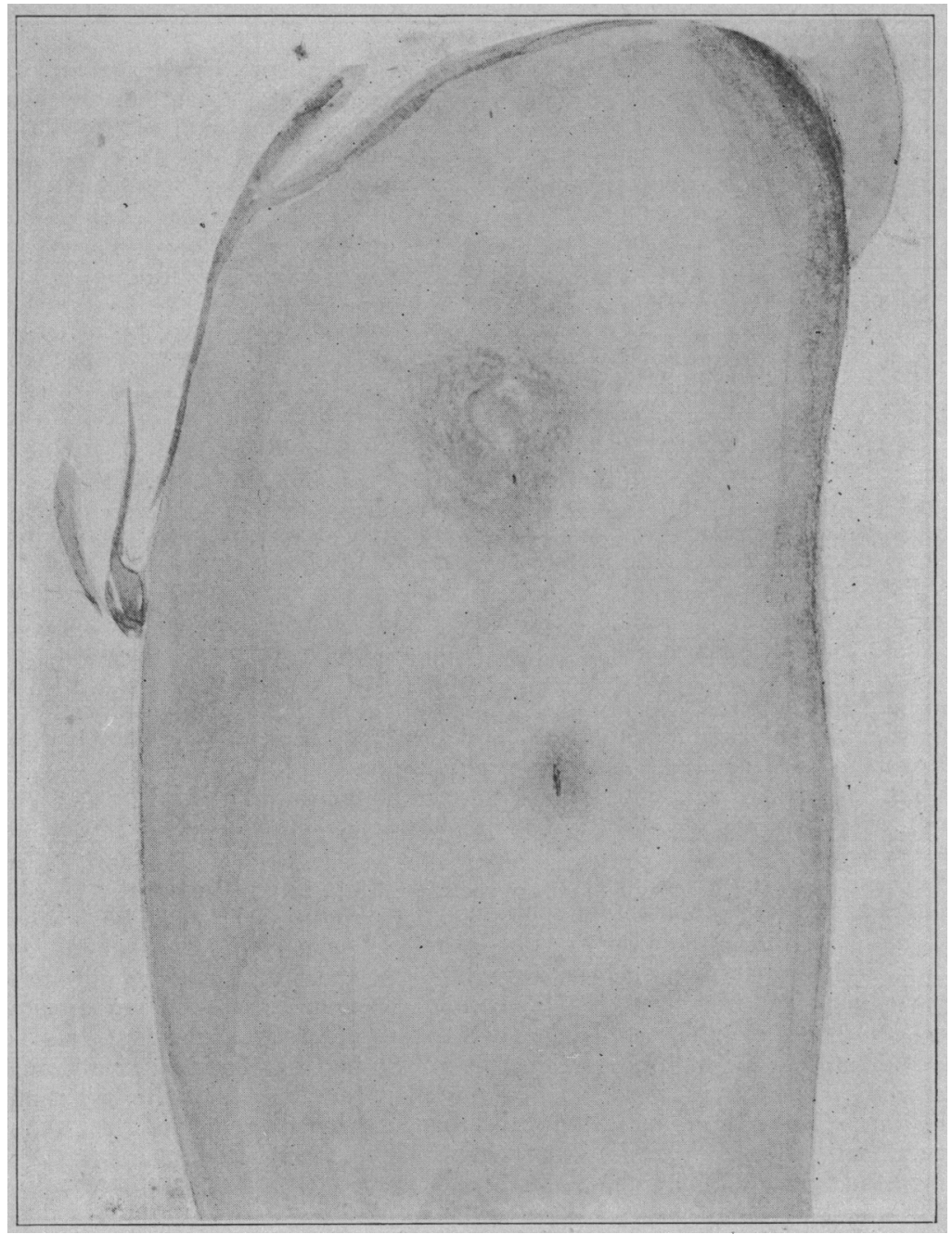

Fig. 2.-Positive intracutaneous reaction (above) made by the intracutaneous injection of $0.02 \mathrm{mg}$. of lactalbumin. The test appeared in less than four minutes, and gave rise to an irregular wheal, which itched slightly and was replaced by a red edematous area of about the same size which persisted for forty-eight hours. A cutaneous reaction (below) made at the same time by the application of powdered lactalbumin to a scarified area on the skin, with addition of a tenth normal solution of sodium hydroxid solution gave rise to no wheal whatever. 
without discomfort and without the appearance of hives. He was then put $0: 1$ a bland diet with gradually increasing quantities of milk, until finally he was able to take $1 \frac{1}{2} 2$ quarts daily without discomfort.

Intracutaneous tests are now (two years later) still positive for lactalbumin and beef albumin.

The subsequent history of the case is of no interest in this connection. The kidney stone passed and the gallstones were removed by operation.

CASE 6.-Female, aged 51, referred by my office associate, Dr. L. S. Milne, had consulted him on account of severe attacks of abdominal pain purpura and angioneurotic edema. Her mother was subject to asthma late in life. Her father and a paternal uncle and aunt were subject to attacks of hives and angioneurotic edema, resembling the attacks complained of by the patient.

Previous Illnesses.-The patient had been subject to asthma fifteen years ago; she had had stomach and intestinal trouble during her entire life, and she had discovered many years ago that she was unable to eat several foods without becoming violently ill. Those of which she was most certain were rice, beef, milk and eggs. She tested herself under a physician's advice by eating a small amount of rice. Within a few moments she experienced a sensation of extreme weakness, followed in less than one-half hour by abdominal pain, which was agonizing, with nusea, vomiting, diarrhea and mucus stools. A few hours later she had an attack of hives, angioneurotic edema and subcutaneous hemorrhages. She believed similar symptoms had been caused on different occasions by the ingestion of meat and milk, but that they had not appeared so quickly.

The history was otherwise uninteresting, except in that several years previously, in search of relief, the appendix and ovaries had been removed. The symptoms were not noticeably changed by this.

Physical examination disclosed several abnormalities which were of little or no interest in this connection; namely, a slight grade of arthritis, irregular pupils, a slight grade of hypertension with cardiac hypertrophy, a slight trace of albumin in the urine, and a suspicious Wassermann reaction.

Cutaneous tests for sensitiveness were made by applying the powdered proteids of milk, egg, rice, asparagus, cabbage, bean and potato to a scarified area on the skin, but gave practically no reaction. Intracutaneous tests, however, made with casein, lactalbumin, egg white and the proteids of rice, cabbage. beans and potato gave rise immediately to wheais from 1 to $2 \mathrm{~cm}$. in diameter, surrounded by large erythematous areas. Within twenty-four hours, the wheals reached the diameter of from 2 to $5 \mathrm{~cm}$. and large areas of angioneurotic edema appeared on 1l:e left hand and hip. After a second twenty-four hours, the wheals disappeared. A few hours later the patient experienced abdominal pain which required one-half grain of morphin for relief. During the attack, there was slight distension of the abdomen, extreme general abdominal tenderness and muscle spasm. The abdominal muscles were held rigidly on account of the pain. The pulse was accelerated, but the temperature and leukocyte counts were normal. Roentgen-ray examination of the stomach during the attack showed the same general features observed at a previous examination when she was free from pain, except for the fact that there was an increase in tone and an increase in the number and depth of the peristalic waves.

A second attack, similar in every detail and in time, was brought on a week later by the injection of $0.01 \mathrm{mg}$. of lactalbumin.

This case of Henoch's purpura probably does not belong exactly in the same clinical group as those previously reported. It is described for the purpose of showing how violent may be the gastric and cutaneous reactions caused by the ingestion or injection of proteins to which an individual is hypersensitive. 


\section{DISCUSSION}

I do not wish in this paper to discuss broadly the subject "allergy." I wish, rather, to confine my discussion and report to the concise fact that a reaction caused by hypersensitiveness to a food can give rise to severe abdominal pain, and that pain may be the prominent, and often the sole striking manifestation of the reaction. The abdominal symptoms of the reaction may be very violent, and can easily be mistaken for those caused by a serious abdominal lesion.

I have observed a number of patients who have been sensitive to one or more of the following foods: egg white, egg yolk, shad roe, lactalbumin, casein, beef, pork, honey, strawberries, lettuce, almonds, beans, onions, cabbage, rice, potatoes, tomatoes, paprica and pimento, and who have invariably had an attack of severe abdominal pain whenever they have eaten the foods to which they were sensitive. In the majority of the cases pain appeared soon after ingestion of the food and lasted from three to six hours. In several cases, however, it did not appear for from three to twenty-four hours, and in these cases, it lasted much longer. In a majority of the cases pain was associated with nausea and vomiting, less frequently with indigestion, bloating, diarrhea, and mucus stools, and much less frequently with hives, angioneurotic edema and purpura.

The pain complained of was evidently the result of a reaction caused by contact between the gastro-intestinal mucosa and the food product to which it was sensitive. This contact gave rise to gastrointestinal symptoms in much the same way that contact of the mucous membrane of the respiratory tract with a pollen to which it is sensitive gives rise to symptoms of hay-fever or asthma. In other words, the pain and other gastro-intestinal symptoms described seem fundamentally analogous in pathogenesis to the symptoms hay-fever and asthma: An attack of asthma can be brought on in a sensitive individual by a subcutaneous injection of the pollen to which he is sensitive. Analogous to this, several of the patients had gastro-intestinal pain after subcutaneous injections of an extract of the food to which they were sensitive.

An experience of interest in this connection might be mentioned here showing that allergy, caused even by the injection of pollen extract in the treatment of hay-fever and asthma, can cause abdominal pain as part of a general reaction. A minority of patients who suffer from asthma and hay-fever complain of dyspeptic symptoms which appear at the beginning of the hay-fever season each year and disappear with the first frost. Occasionally, such a patient experiences abdominal pain in addition to the ordinary dyspeptic symptoms. One such patient, while under treatment with pollen extract, experienced abdominal pain 
after each inoculation of pollen, which was so severe that he refused treatment after the third inoculation on this account. The pain was severe, steady, nonradiating and localized in the epigastrium. It appeared a few minutes after each inoculation and lasted several hours. It was not accompanied by other symptoms of allergy; in fact, in this case it was the sole manifestation of reaction to the pollen.

It is interesting to mention that among the patients having food allergy observed by me, nearly 50 per cent. had demonstrable pathologic lesions in the alimentary tract or in its appendages. These lesions were recurrent appendicitis, gallstones, duodenal ulcer, dense adhesions and extreme ptosis. Food allergy, like other forms of allergy, is, perhaps, primarily dependent on an inherited constitution which renders the individual susceptible of becoming hypersensitive to certain alien substances. It seems quite possible, however, that an abnormality in the alimentary tract may be a contributory factor in the etiology of this type of case.

It is furthermore of interest to note the fact that in two cases attacks of acute appendicitis, which required surgical intervention, followed attacks of food allergy. While it is hardly justifiable to draw conclusions from two cases, it is justifiable to say that alimentary allergy is a very real cause of gastro-intestinal turmoil. It may cause violent muscular contractions, pathologic secretions, edema of the mucous membrane and local anemia. It is very reasonable to suppose that this may occasionally set up infection and inflammation and give rise to serinus disease in the alimentary tract or in its appendages.

DIAGNOSIS

In the diagnosis of alimentary allergy as the cause of abdominal pain, the history of the case is all important. If a patient invariably has pain and other gastro-intestinal symptoms after eating certain articles of food, and is at other times free of digestive disturbances, the diagnosis is simple and easy. If, with this, there should be other manifestations of allergy, such as hives, angioneurotic edema, or asthma, the diagnosis is comparatively certain. It can further be verified in the great majority of cases by the use of intracutaneous tests with the suspected proteids. Skin tests in this particular type of allergy are not always so clearly positive as they are in hay-fever and asthma, but in almost every case characteristic wheals can be obtained if intracutaneous tests are made with the correct proteins.

In making a diagnosis of food allergy it is not advisable to accept a patient's statement for the fact that the eating of a certain food invariably causes abdominal pain. Patients are often mistaken about this. It will often be found on putting them to a test and having them eat the suspected food that it causes no disturbance whatever.

When an individual is hypersensitive to an uncommon article of diet, such as shad roe or honey, the diagnosis of the condition is 
easy and is often made by the patient himself. He usually gives a history of occasional attacks of pain and vomiting, which last a few " hours, and usually states that he is free of digestive disturbance at other times. When he is sensitive to a common food, such as eggs or milk, however, the diagnosis may be very difficult. Milk and eggs are used so commonly in cooking, that a patient who is highly sensitive to either one may be in a reactive state almost constantly, and may have such symptoms as pain after eating, nausea, vomiting, bloating and indigestion almost every day. He may be unable to place the blame on any one particular food. The diagnosis may be doubly difficult because of the fact that the gastro-intestinal mucous membrane, when in a chronically reactive state, is often very irritable, with the result that rough, or stimulating, foods, such as coarse vegetables, fruits, nuts, condiments, alcohol, etc. irritate the stomach and augment the symptoms. This usually leads a patient to place the blame for his trouble on foods of this variety rather than on the primary offenders, milk or eggs. It is in cases of this type that cutaneous tests have their greatest sphere of usefulness. One can occasiona!ly discover the offending proteid by having the patient eliminate first one and then another common article of diet for a period of a week or more. By keeping careful notes, he may be able to discover on which diet he is most comfortable and then by eating a suspected food determine whether or not it actually causes distress. This method of diagnosis may be very slow, however, and may even fail utterly should the patient be sensitive to two or more common articles of food.

\section{CLTANEOCS TESTS}

Cutaneous tests are not essential to the diagnosis of food allergy when an individual is sensitive to one unusual article of diet, such as honey or shad roe, and has pain soon after the eating of certain foods. In cases of this sort, the diagnosis can usually be made without a cutaneous reaction. The great sphere of usefulness for cutaneous tests is in patients who are sensitive to a common article of diet, such as milk or eggs, or who are sensitive to several articles of diet, or for patients who have delayed reactions and experience no discomfort for a number of hours after the ingestion of a food. For the correct and prompt diagnosis of cases of this type cutaneous tests are indespensable.

Several methods of making cutaneous tests are now being used. A method described by Walker is the simplest and most convenient. It consists of applying the suspected proteids in dry powdered form to a scarified area on the skin and placing on this a small drop of tenth normal sodium hydroxid solution. The appearance of a wheal indicates a positive reaction. 
This method is very satisfactory in the majority of hay-fever and asthma cases. It failed almost completely, however, in a number of cases of food allergy observed by me which gave very large wheals after intracutaneous injections of a solution of the suspected proteids. Intracutaneous tests are not so handy as the scarification method because of the fact that test solutions tend to lose their potency and it is quite difficult and laborious to keep a large number of potent solutions on hand. I have used a very simple means of making intracutaneous tests. It can be carried out accurately without much trouble or expense.

\section{INTRACUTANEOUS METHOD}

Place about $0.1 \mathrm{mg}$. of each of the suspected proteids (in dry powdered form) to be used in the tests on a large sterile plate. One-tenth mg. of a powdered protein can be guessed at crudely as the amount of powder which can be taken up easily on the end centimeter of a Peerless round wood applicator. To each proteid add 0.1 c.c. of physiologic sodium chlorid solution (distilled water will not dissolve globulin). Stir each carefully with separate wooden applicators until the proteids go into solution. Draw up the first solution to be used in a tuberculin syringe armed with a small needle, and inject intracutaneously 0.02 c.c. ( $0.02 \mathrm{mg}$. of proteid). Wash the syringe carefully in each of three separate vessels containing sterile physiologic sodium chlorid solution, numbered 1,2 and 3 . If care is used, the last washing should remove every trace of proteid used in the previous test. The same syringe may then be used for the second test. and the same wash waters can be usd several times, that is, if the vessels are always used in rotation according to their numbers $-1,2$ and 3 .

This method has several advantages. First, it is likely to give a larger number of definite positive reactions than scarification tests. Second, dry proteins keep indefinitely. Third, it is simple.

The scarification and intracutaneous methods are compared in Figures 1 and 2. Figure 1 represents a patient (Case 1 ) on whom the two methods gave exactly the same result. Figure 2 represents a patient (Case 6) on whom a cutaneous test was practically negative, whereas an intracutaneous test gave rise immediately to a definite wheal.

In a normal person nothing striking follows an intracutaneous injection of a foreign protein. If an individual is inoculated with a proteid to which he is sensitive, however, in a few moments a wheal will usually appear which varies in size from 0.5 to $2 \mathrm{~cm}$. or more. in diameter. The wheal is usually paler than the skin, usually shows pseudopod-like projections, is often surrounded by an irregular erythematous area, and often itches intensely.

The wheal usually appears and reaches its minimum size within a few minutes. It may not reach its maximum size for several hours, however. If it appears quickly, it usually disappears within one or two hours. If it reaches its maximum more slowly, it usually lasts much longer. 
Cutaneous tests are not so striking in the average case of food allergy as they are in patients who have hay-fever and asthma. In the pollen cases, the scarification method is almost always adequate, and, as a rule, gives rise to a large wheal within a few minutes if the correct pollen is used. In food cases, however, an intracutaneous test is very often necessary for a convincing reaction.

TREATMENT

The treatment of food allergy may be considered from three points of view. First, removal of the cause; second, protein treatment; third, symptomatic treatment.

Removal of the cause is very simple when a person is sensitive to a rare article of food, such as honey, shad roe, strawberries, beans or paprica. Nothing is easier than to instruct a patient to cease eating such articles and to live in comfort. When an individual is sensitive to a food used so commonly in cooking as eggs or milk, however, removal of the cause is by no means easy. Unfortunately, a person is often so highly sensitive that a mere trace of the food to which he is sensitive suffices to make him miserable. He may find it necessary to be constantly on the lookout to avoid taking such a trace unknowingly. For this reason, protein therapy in a case of this sort is advisable. It must be mentioned here that in treating a case of this type, it is not necessary to obtain complete desensitization in order to relieve the patient. Complete desensitization may not be possible, and an effort to attain this result may not be advisable or really very desirable. If a patient's sensitiveness is reduced to a point where he can simply tolerate the small amounts of the food which are used in cooking other foods, he can, with a little care, live a life of comfort. For example, if a patient were sensitive to casein, and his sensitiveness were reduced to such a point that he could tolerate the amount of casein encountered in ordinary cooked foods, such as bread, cake, potatoes, etc., he could, by avoiding the drinking of milk and the eating of foods consisting chiefly of milk, live in comfort. It is not possible here to discuss broadly the subject of protein therapy. For this the many splendid articles already published should be consulted.

As symptomatic or temporary measures might be mentioned a bland diet, such as that used for hyperacidity, avoiding, of course, the proteins to which the patient is sensitive; sodium bicarbonate, in doses sufficient to reduce hyperacidity; atropin, and in the severer cases, removal of the exciting agent by gastric lavage and purgation and epinephrin or morphin subcutaneously, if needed.

\section{SUMMARY AND CONCLUSION}

It is well known that humans and animals may become sensitive to alien bodies of many varieties. When this is the case, they react when- 
ever they come in contact with the body to which they are sensitive. The gastro-intestinal mucosa may become hypersensitive to an article of food with the result that the patient experiences severe abdominal pain, often associated with nausea and vomiting, whenever he eats the food to which he is sensitive. These alimentary symptoms are in many cases the sole striking manifestations of the reaction. Individuals sensitive to uncommon articles of diet, such as shad roe, lettuce, honey, strawberries, cabbage, tomatoes, paprica, etc., usually give a history of occasional attacks of abdominal pain and digestive upset. They are, as a rule, free of digestive disturbance between attacks. Individuals sensitive to the commoner articles of food, such as milk or eggs, have more frequent attacks of pain and are often subect to chronic indigestion as well.

The symptoms of food allergy may simulate somewhat those of a surgical lesion in the abdomen, and an error in diagnoses can be made unless this condition is kept in mind.

Food allergy was observed more frequently in individuals who had organic lesions in the gastro-intestinal tract or in its appendages than it was in normal persons.

Food allergy had acute appendicitis as a sequel in two cases, and surgical intervention was required.

\section{BIBLIOGRAPHY}

Arthus, M.: Injections Répétées de sérum de cheval ches le lapin, Compt. rend. Soc. de biol. Par. 55:817, 1903. Anaphylaxie et immunité, Compt. rend. Acad. d. sc., Par. 154:1363, 1912. Anaphylaxie passive du lapin, Compt. rend. Soc. de Biol. 82:412, 1919.

Besredka, A., and Gloyne, S. R.: Anaphylaxis and Anti-Anaphylaxis and Their Experimental Foundation, St. Louis, C. V. Mosby Company, 1919.

Blackfan, K. D.: A Consideration of Certain Protein Hypersensitiveness in Children, Am. J. M. Sc. 160:341, 1920.

Boughton, T.: Anaphylaxis and Allied Phenomena in Relation to Disease, J. Lab. \& Clin. M. 5:597, 1920.

Boughton, T. H.: Studies in Protein Intoxication, J. Immunol. 1:105, 1916; 2:501, 1917; 4:213, 1919. 1920.

Coca, A. F., and Kosakai, M.: Studies in Anaphylaxis, J. Inrmuno1. 5:297,

Coca, A. F.: Mechanism of Anaphylaxis Reaction in Rabbits, J. Immunol. 4:219, 1918. Hypersensitiveness: Anaphylaxis and Allergy, J. Immunol. 5: $363,1920$.

Cooke, R.: Protein Sensitization in the Human, with Special Reference to Bronchial Asthma and Hay-Fever. M. Clin. N. America 1:721, 1917.

Engman, M. F., and Wander, W. G.: Application for Cutaneous Sensitization to Diseases of the Skin. Arch. Dermat. \& Syph. 3:223, 1921.

Gay, F. P., and Southard. E. E.: On Serum Anaphylaxis in the GuineaPig, J. M. Research 16:143, 1907; 18:407, 1908; 19:1, 5, 17, 1918.

Joltrain: Treatment of Alimentary Anaphylaxis, Bull. et. mém Soc. méd. d. hôp. de Par. 43:556, 1919.

Lowdermilk, R. C.: Hay-Fever, J. A. M. A. 63:141 (July 11) 1914.

Laroche, G., and Richet. C.: L'anaphylaxie alimentaire état actuel de la question, Bull. méd. Par. 34:625, 1920. 
McBride, W. L., and Schorer, E. H.: Erythematous and Urticarial Eruptions Resulting from Sensitization to Certain Foods, J. Cutan. Dis. 34:70, 1916. Novy, F. G., and DeKruif, P. H.: Anaphylatoxin and Anaphylaxis, J. A. M. A. 68:1524 (May 26) 1917.

Otto, R.: Das Theobald Smithsche Phaenomen der Serum-Ueberempfindlichkeit, Gdnkschr. f. d. Verstorb. Generalstabsarzt d. Armee 1:153, 1906.

Von Pirquet, C., and Schick, B.: Theorie der Inkubationszeit, Wien. klin. Wchnschr. 16:753, 1903.

Pagniez, P., and Vallery. R.: Digestive Anti-Anaphylaxis, Ann. méd., Par. 8: 303,1920 .

Rosenau, M. J., and Anderson, J. F.: A Study of the Cause of Sudden Death Following the Injection of Horse Serum, U. S. Hygienic Lab. Bull., No. 29, 1906. Studies on Hypersusceptibility and Immunity, ibid., No. 36, 1907. Further Studies on Anaphylaxis, ibid., No. 45, 1908.

Rosenau, M. J.: Studies in Food Poisoning: An Experimental Lunch with Canned Food Containing Bacteria, M. Clin. N. America 3:913, 1920.

Rosenau, M. J., and Anderson, J. F.: Hygienic Lab. Bull., No. 29, 1906; No. 36, 1907; No. $45,1908$.

Vaughan, V. C.: Protein Split Products in Relation to Immunity and Disease, Philadelphia, Lea \& Febiger, 1913.

Vaughan, V. C., and Wheeler, S. M.: The Effects of Egg-White and Its Split Products on Animals; a Study of Susceptibility and Immunity, J. Infect. Dis. 4: $476,1907$.

Walker, I. C.: Studies on the Cause and Treatment of Bronchial Asthma, J. A. M. A. 69:363 (Aug. 4) 1917. Causation of Eczema, Urticaria and Angioneurotic Edemaby Proteins Other Than Those Derived from Foods. Study XVIII, J. A. M. A. 70:897 (March 30) 1918. Bronchial Asthma, Arch. Int. Med. 22:466 (Oct.) 1918. Studies on the Sensitization of Patients with Bronchial Asthma, Studies I-V, J. M. Research 35:373, 391, 487, 497, 309, 1917 ; Studies VI-VIII, J. Immunol. 2:227, 237, 243, 1917; Study IX, Am. J. Bot. (July) 1917; Studies X-XIV, J. M. Research 36:231, 237, 245, 423, 1917; Studies XV-XVII, ibid. 37:57, 377, 287, 1917.

Wells, H.: The Present Status of the Problems of Anaphylaxis, Physiol. Rev. 1:44, 1921.

White, C. J.: Two Modern Methods to Be Employed in the Treatment of Chronic Eczema, J. A. M. A. 68:81. 1917.

Zinsser, H.: The More Recent Developments in the Study of Anaphylactic Phenomena, Arch. Int. Med. 16:223 (Aug.) 1915. 